\title{
EXCRETION OF CATECHOLAMINES, 17-KETOSTEROIDS, 17-HYDROXY- CORTICOIDS AND 5-HYDROXYINDOLE IN MEN EXHIBITING A PARTICULAR BEHAVIOR PATTERN (A) ASSOCIATED WITH HIGH INCIDENCE OF CLINICAL CORONARY ARTERY DISEASE * $\dagger$
}

\author{
By MEYER FRIEDMAN, SHIRLEY ST. GEORGE, SANFORD O. BYERS AND
} RAY H. ROSENMAN

(From the Harold Brunn Institute, Mount Zion Hospital and Medical Center, San Francisco, Calif.)

(Submitted for publication August 26, 1959; accepted January 8, 1960)

Several recent studies from this laboratory strongly suggest that a particular overt behavior pattern may be strongly implicated in the pathogenesis of many cases of clinical coronary artery disease. Thus it was observed (1) that when accountants were forced by occupational circumstances to alter acutely their usual behavior pattern of relative calm to an overt behavior pattern in which competitive and excessive drive and an enhanced sense of time urgency were paramount, a marked elevation of serum cholesterol and hastening of blood clotting time occurred. It is of interest that this observed rapid rise of serum cholesterol following change in behavior pattern already has been confirmed by three different groups of investigators (2-4). It was further found (5) that a group of individuals who chronically exhibited an overt behavior pattern (Pattern A) characterized by excessive and competitive drive and an enhanced sense of time urgency not only exhibited notably higher serum cholesterol and faster clotting time than individuals exhibiting a converse type of behavior pattern (Pattern B), but also an approximately sevenfold higher incidence of clinical coronary artery disease and over three times as much arcus senilis. Finally, we have more recently found (6) that females exhibiting this same Behavior Pattern A exhibit approximately the above same differences in blood and cardiovascular findings, when compared to females showing Pattern B. These women with

* Supported by the Life Insurance Medical Research Fund, National Heart Institute, National Institutes of Health H-119, American Heart Association, and Alameda County Heart Association.

$\dagger$ Read in part before the Annual Meeting and 32nd Scientific Sessions of the American Heart Association, Philadelphia, Pa., October 25, 1959.
Pattern A appeared to be remarkably similar to men with Pattern A as regards the incidence and intensity of their blood and cardiovascular abnormalities.

Since the dietary, physical activity and various other characteristics of these different types of individuals were approximately the same, we concluded that in some manner Behavior Pattern A itself was causally responsible for the altered lipid and clotting dynamics of the blood and for the clinical changes found. The question of how such a behavior pattern might induce such changes remained to be clarified. As a first attempt to ferret out possible clues, it was decided to study the urinary excretion of some possibly relevant hormones in groups of men exhibiting Behavior Pattern A or Pattern B. The results of this study are presented below.

\section{METHODS}

Choice of men and method of urine collection. In our earlier study (2) a large group of men had been chosen for us (by lay selectors) who exhibited either Behavior Pattern A or B. In our own personal interview, we also had the opportunity to judge for ourselves the intensity of the pattern present in each volunteer subject. We therefore selected 12 men (between the ages of 35 and 50 years) from each of these two groups who we believed possessed most completely the characteristics of the respective two behavior patterns.

Earlier we expressed our belief (5) that Behavior Pattern A might be a resultant of the interplay between a provoking environment and a particular type of personality. We therefore employed three general criteria in selecting the 12 men of Group A. First, each of the 12 men occupied a position not necessarily of top executive quality but associated with or demanding extreme competitive activity and deadline preoccupations. Thus, of the 12 men selected, 4 were managing or city editors; 7 were corporation executives who were themselves 
directly engaged in highly competitive merchandising or engineering activities; and one was an advertising account executive supervising a particularly difficult account.

Second, each of the men chosen admitted the chronic presence of a rather marked sense of competitive drive and most particularly a severe sense of time urgency.

Third, and most important, all of the men had exhibited various motor phenomena during our interview with them suggesting the presence of a severe sense of time urgency. These phenomena were 1) a rapid, frequently forced and audible intake of breath, 2) explosive intonation or emphasis of individual words of sentences, 3) tense facial and body musculature, 4) frequent fist clenching accompanying ordinary conversation, 5) consistent tendency to hasten the pace of ordinary conversation either by attempting to finish the sentences of the interviewer or to hasten the latter's rate of speaking by uttering rapidly "yes, yes" or "I see, I see" even before the interviewer had finished his sentence.

The converse of these three criteria was used to select 12 men having the $B$ Pattern. Their positions demanded neither competitive activity nor preoccupation with deadlines. Thus of the 11 selected men who agreed to participate, 8 were municipal employees performing clerical or accounting duties; 2 were employee embalmers; and one was an employee public accountant.

None of the men of either group was ever aware of suffering from symptomatic clinical coronary artery disease. All were normotensive.

If a particular behavior pattern might alter the secretion, hence also the excretion rate of hormones to be studied, obviously the products of excretion should be collected at the time that the particular behavior pattern might be most expected to be present. Each subject, therefore, was requested to collect all of his urine while at work. We obtained approximately half of the day's sample from 10:00 a.m. to 12:00 noon and the remainder from 2:00 to 5:00 p.m. Urine voided on reaching work at 9:00 a.m. and after returning from lunch at 2:00 p.m. was discarded. Urine was thus collected separately from each subject on each of four successive working days (Monday to Thursday, inclusive), but then was pooled and analyzed as described below. In addition, a single overnight urine sample was obtained from each subject immediately upon arising. We thus were able to obtain urine formed during the height of their working period and also during complete bed rest. Each man also was asked to estimate the intensity of his sense of urgency during the four days of urine collection. The men were cautioned not to ingest bananas during the four day period of urine collection. None was taking any type of medication. The urines of four men (two of Group A and two of Group B) were obtained each week.

Urinary assay of 17-ketosteroids, 17-hydroxycorticosteroids, 5-hydroxyindole, epinephrine and norepinephrine. In all of the chemical assays listed below, a urine from a man of Group A was always analyzed in the same run with a urine obtained from a man of Group B.
The creatinine content of each four day pooled and single night specimen of urine was determined by the method of Bonsnes and Taussky (7). The 17-ketosteroid content of the same specimens was determined according to the method of Klendshoj, Feldstein and Sprague (8). The 17-hydroxycorticosteroid analysis was made according to the method of Silber and Porter (9). The 5-hydroxyindole analysis was done according to the method of Dalgliesh (10). The epinephrine and norepinephrine content of the urine samples was determined according to the method of Sobel and Henry (11), as modified by Forsham (12). This modification consisted of the addition of $1 \mathrm{ml}$ of 2 per cent ascorbic acid and $1.5 \mathrm{ml}$ of 1 per cent ethylenediaminetetraacetate disodium to $15 \mathrm{ml}$ of hydrolyzed urine aliquot just prior to adjusting $\mathrm{pH}$ to 8.4 and chromatographing on $\mathrm{Al}_{2} \mathrm{O}_{3}$. Aliquots of the acetic acid eluate were oxidized with ferricyanide at $\mathrm{pH} 3.5$ and 6.0 and stabilized with additional alkaline ascorbate. In our hands, the standard deviation of individual values in replicate analyses was \pm 8 per cent for norepinephrine and \pm 10 per cent for epinephrine. Recovery of added epinephrine ranged between 90 and 94 per cent, and of added norepinephrine, between 88 and 94 per cent.

The concentrations of these substances were expressed in terms of milligrams or micrograms per gram of creatinine, for comparative purposes.

Study of dietary, tobacco, alcohol, work and exercise habits. These data already had been obtained in these men in 1958 (2), and as far as we could determine there had been no change of any consequence in their habits except that one individual (W.L. of Group A) had given up smoking cigarettes.

Study of serum cholesterol, blood clotting, and incidence of arcus senilis and clinical coronary artery disease. These data also had been obtained in these men in 1958 (2). Serum cholesterol determinations were repeated in 6 of the 23 men, and as Table I indicates, there was no essential change from the values obtained in these same men in 1958.

\section{RESULTS}

General characteristics of the two groups. As Table I indicates, the age, weight and height of the two groups of men were approximately the same. The hours of working and the average amount of physical activity were greater in men of Group A. However, the average excess physical activity of the men of Group A was due to their indulgence in weekend sports. During the weekday period of urine collection, however, these men indulged in no more, but probably in less, physical activity than the men of Group B. Once again (2) the cigarette and alcohol consumption were found to be considerably greater in men of this same group. 
FRIEDMAN, ST. GEORGE, BYERS AND ROSENMAN

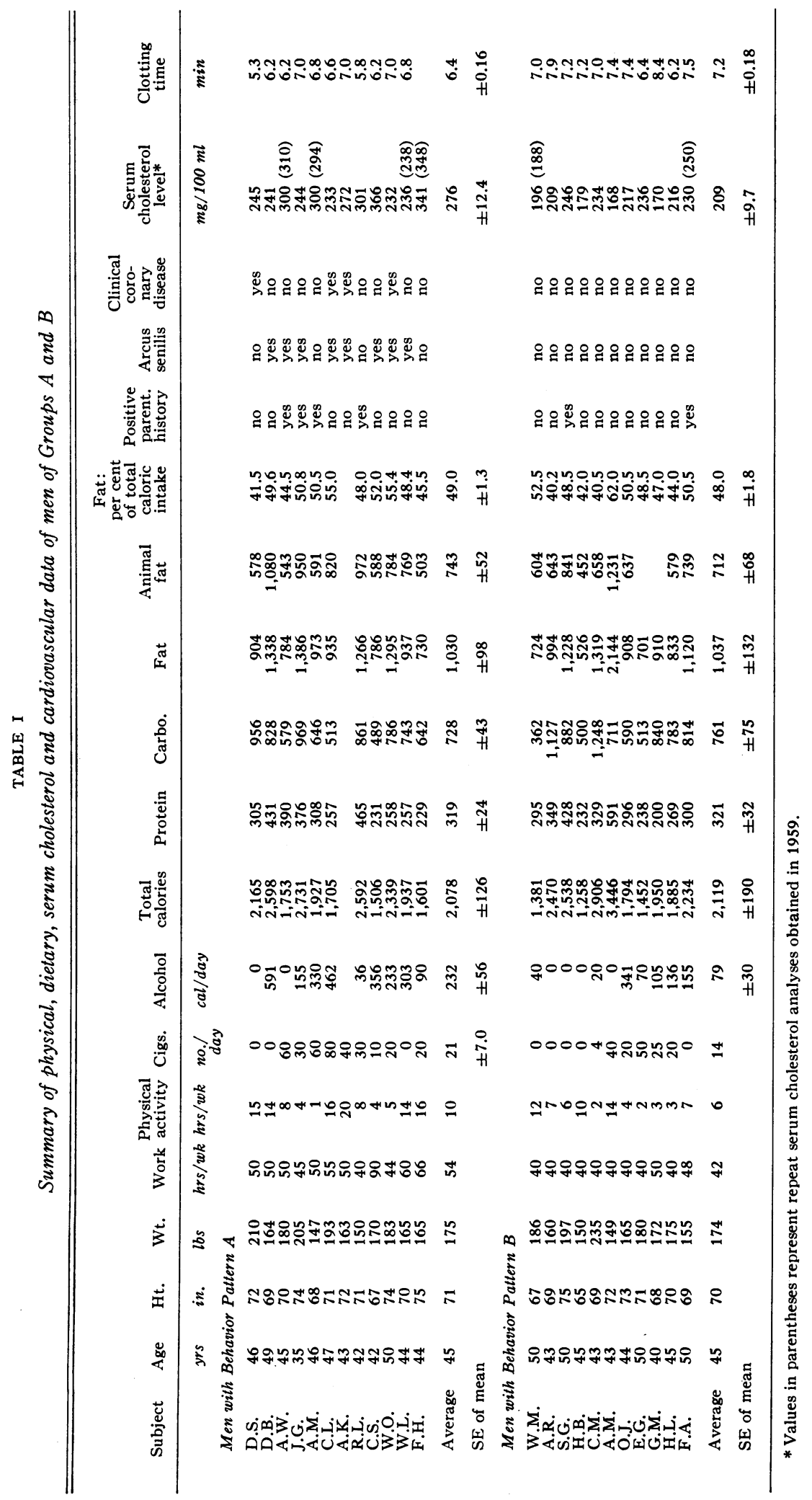


HORMONAL EXCRETION IN OVERT BEHAVIOR PATTERN A

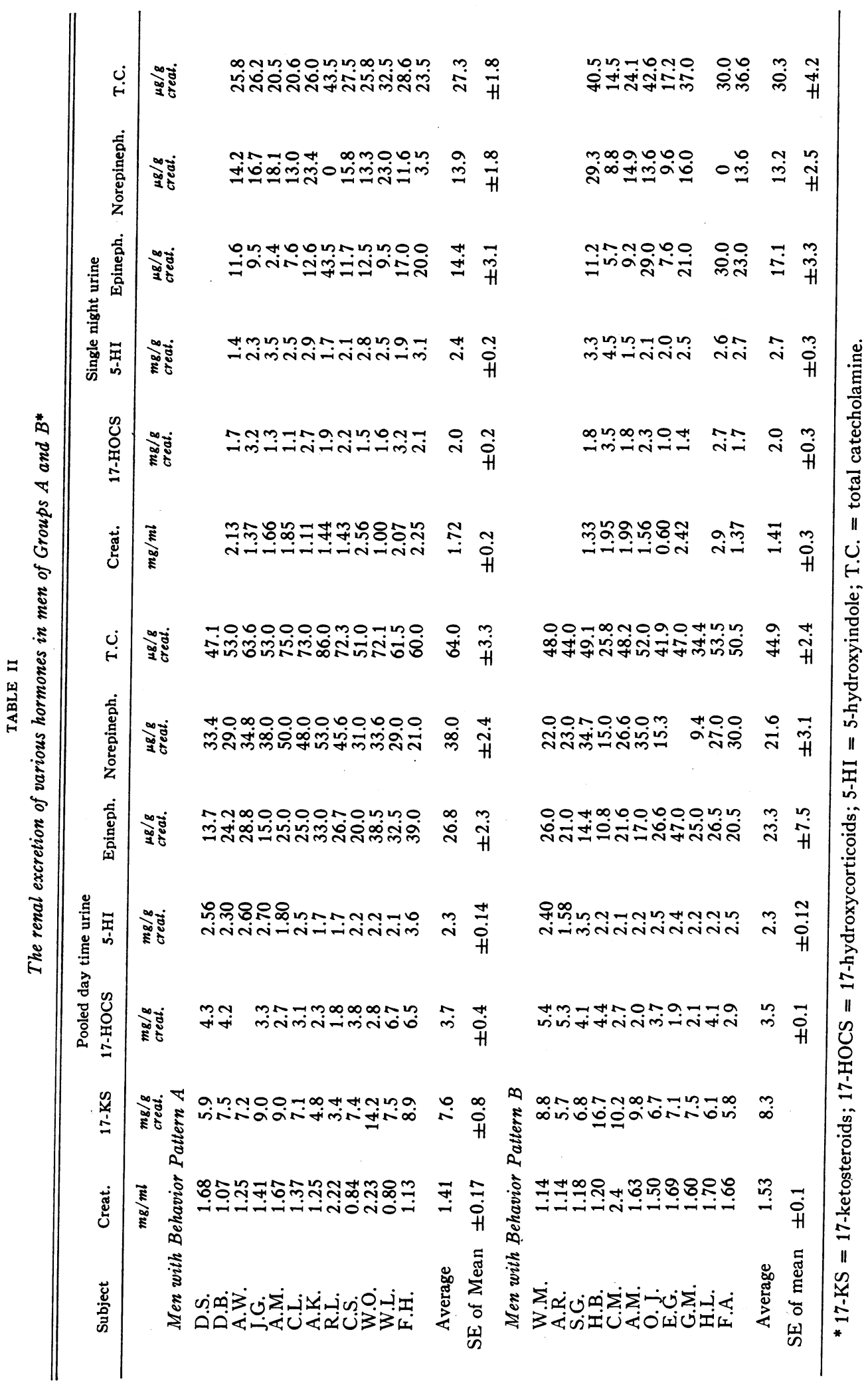


The dietary habits of the two groups. The intake of total calories, protein, carbohydrate and fat (also animal fat) was essentially the same in both groups (see Table I). Similarly the per cent of the total diet ingested as fat was approximately the same.

Serum cholesterol and clotting time of the two groups. As Table I shows, despite the small number of subjects involved, the average serum cholesterol of the men of Group A (276 mg per 100 $\mathrm{ml}$ ) is remarkably and significantly higher than that of Group B. Again, the clotting time is significantly faster in the men of Group A than in the men of Group B. These average differences in serum cholesterol and blood clotting time were greater than those noted in the earlier study because, we believe, the extreme examples of both types of behavior pattern were chosen for the present study.

The incidence of arcus senilis and clinical coronary artery disease. The previously observed striking differences in incidence of arcus senilis and clinical coronary disease again were found. Thus 8 of the 12 men of Group A, but none of the men of Group B, displayed an arcus senilis. Similarly, four of the men of Group A and none of Group B were found to have clinical, but asymptomatic coronary artery disease. The evidence for the latter's presence was electrocardiographic in each case. Two of the four electrocardiograms, besides exhibiting characteristic patterns of myocardial damage, also indicated the occurrence of old infarcts that had escaped clinical recognition.

The renal excretion of various hormones in the two groups. Eleven of the 12 men of Group A reported that the pooled daytime urine was collected while they were feeling a moderate to severe sense of urgency doing their job. On the other hand, 7 of the 11 men of Group B reported feeling no sense of urgency during the daytime urine collection. The remaining four felt only a slight to moderate sense of urgency. It was interesting that the renal excretion of the various hormones studied was approximately the same (see Table II) when both groups were at bed rest. However, quite significant differences appeared during the working periods of the two groups.

In both groups, the excretion of 17-hydroxycorticoids increased comparably during the work- ing periods. This observed higher daytime excretion of 17-hydroxycorticoids in almost all the subjects confirms the earlier observation of Laidlaw, Jenkins, Reddy and Jakobson (13). This finding also suggests that each of the subjects had carefully followed our directions for the separate collections of the two types of urine. The urinary excretion of 17 -ketosteroids during the working period also was approximately the same in the two groups. The excretion of 5-hydroxyindole also was found to be approximately equal both during the night and during working periods in the two groups.

A marked increase in the excretion of epinephrine occurred during the working period of both groups. Although an increase of 86 per cent occurred in the men of Group A and 36 per cent in the men of Group B, the difference was not statistically significant. However, the increase in the excretion of norepinephrine during the day (173 per cent) shown by the men of Group A was of much greater magnitude than the increase (64 per cent) observed in the men of Group B and this difference was significantly different (difference of means $=4.2 \times \mathrm{SE}$ difference of means). The daytime excretion of the total unchanged catecholamines by the men of Group A also was significantly greater than that by the men of Group B (difference of means $=4.7 \times \mathrm{SE}$ difference of means).

As Table II indicates, no correlation was found between the average weekly amount of physical activity done by men of either group and their norepinephrine secretion. This absence of correlation we believe was due to the fact that the greater amount of physical activity reported by a number of individuals was due not to the demands of their job but to the propensity of these individuals to indulge in sports during the weekend -a period in which the urine collections were not obtained.

\section{DISCUSSION}

Earlier (2) we stressed our belief that Behavior Pattern A was a resultant of the interaction of two variables, namely, the specific reaction of an individual to his given environment, and the quality and intensity of the inciting or provocative environment itself. So composed, a change 
either in the individual's reaction (endogenous) or in the inciting environment (exogenous) easily could modify or even conceivably eliminate the pattern.

Because of this, we felt that any study of the possible biochemical consequents of Behavior Pattern A should be conducted precisely during the individual's exposure to that milieu most likely to evoke it. The present study indicates rather clearly, we believe, that when such precautions were taken, individuals exhibiting Pattern A excreted far more norepinephrine than subjects exhibiting the converse pattern. However, when the specific milieu was not provoking (i.e., bed rest), the catechol excretion of both groups of men was essentially identical.

These findings suggest that the excess catechol excretion by the men exhibiting Pattern $\mathrm{A}$ is not a fixed but rather a phasic phenomenon apparent only when they are studied in the appropriate environment. Indeed, if only 24 hour urine collections had been made, we seriously doubt if the differences found during the working period would have been sufficiently great to offset the probable equivalence of values obtained during the remaining 16 to 18 hours of the total 24 hour period. We stress these matters because if the present results are to be confirmed, the urine must be collected when each type of behavior pattern is in operation.

A good illustration perhaps of the importance of securing the proper conditions for the study of catecholamine discharge was the finding of Raab and Gigee $(14,15)$, confirmed later by Gazes, Richardson and Woods (16), of a marked increase in the plasma catecholamine of patients with angina after exercise. Yet both groups of authors were unable to observe a similar increase of catecholamine either in the urine or in the plasma of patients convalescing from acute infarction while they were at bed rest. Indeed, it is unlikely that patients who had suffered from a clinically recognized infarction would later exhibit excess discharge of catecholamine since such patients, whether of their own volition or guided by medical advice, often radically alter either those facets of their own personality or those particular environmental factors, the preinfarction combination of which may have induced Behavior Pattern A. Certainly we do not believe that this behavior pattern is an unalterable, psychophysiological status.

Can a phasic excess discharge of catecholamines occurring day in and day out over many years be responsible for the lipid, clotting and cardiovascular abnormalities to which men exhibiting Pattern $\mathrm{A}$ are so prone $(1,5)$ ? Or is this phenomenon too, as are possibly the serum cholesterol elevation, the excessive cigarette smoking and alcohol ingestion, only a congener of the pattern, bearing seemingly (5) little relevance to the pathogenesis of the clinical coronary artery disease so frequently found in men with this pattern? We strongly suspect that this excess catecholamine discharge may play more than such an associative role, for the following reasons. First, the marked increase in incidence of arcus senilis and coronary artery disease previously found (5) in men with Behavior Pattern A could not be ascribed to any excess ingestion of fat, tobacco or alcohol, or solely to the elevated serum cholesterol also observed in men with this pattern. Second, the behavior pattern has those qualities of excessive competitive drive and time urgency already shown by others $(17,18)$, as well as by us, to induce excess catecholamine discharge. Such behavioral characteristics, moreover, are not found in patients with anxiety neurosis, nor have the latter been found to excrete excess catecholamines (19). Third, excess catecholamine has been shown to be capable of altering lipid dynamics (20-23) and perhaps in the direction in which they were observed in men with Behavior Pattern A. Finally, administration of catecholamine markedly worsens the severity of the atherosclerosis occurring in laboratory animals fed excess cholesterol $(24,25)$.

Tempting as the above hypothesis may be, it must tentatively remain only a hypothesis. It would appear, however, that the present study has clearly demonstrated at least one biochemical pathway by which a psychological process may enter into and possibly influence man's more tangible, hence measurable, metabolic functions. It would now appear even more important that certain psychological processes and their resultant biochemical changes not be neglected in future studies of human coronary artery disease, as has so frequently been done in the past. 


\section{SUM MARY}

The urinary excretion of 17-ketosteroids, 17 hydroxycorticosteroids, 5-hydroxyindole, epinephrine and norepinephrine was measured in a group of 12 men exhibiting an overt behavior pattern (A) found in earlier studies to be associated with a high incidence of clinical coronary artery disease. The nocturnal urinary excretion of these hormones was found to be the same in these men as in control men exhibiting a converse behavior pattern. However, when urine formed during working hours was analyzed, it was found that a far greater increase in norepinephrine occurred in the urine of the men exhibiting Pattern A than in the control groups. The possible relevance of these findings to the pathogenesis of clinical coronary disease was discussed.

\section{ACKNOWLEDGMENTS}

The authors wish to express their thanks for the technical assistance of Veronica Mena, Judith Levy, Vernice Carroll, Warren Hayashi and Clarence Omoto.

\section{REFERENCES}

1. Friedman, M., Rosenman, R. H., and Carroll, V. Changes in the serum cholesterol and blood clotting time in men subjected to cyclic variation of occupational stress. Circulation 1958, 17, 852.

2. Wertlake, P. T., Wilcox, A. A., Haley, M. I., and Peterson, J. E. Relationship of mental and emotional stress to serum cholesterol levels. Proc. Soc. exp. Biol. (N. Y.) 1958, 97, 163.

3. Thomas, C. B., and Murphy, E. A. Further studies on cholesterol levels in the Johns Hopkins medical students: The effect of stress at examinations. J. chron. Dis. 1958, 8, 661.

4. Grundy, S. M., and Griffin, A. C. Effects of periodic mental stress on serum cholesterol levels. Circulation 1959, 19, 496.

5. Friedman, M., and Rosenman, R. H. Association of specific overt behavior pattern with blood and cardiovascular findings. J. Amer. med Ass. 1959, 169, 1286.

6. Rosenman, R. H., and Friedman, M. Association of a specific overt behavior pattern in women with increased blood cholesterol and clotting time, arcus senilis, and incidence of clinical coronary disease (abstract). Circulation 1959, 20, 759.

7. Bonsnes, R. W., and Taussky, H. H. On the colorimetric determination of creatinine by the Jaffé reaction. J. biol. Chem. 1945, 158, 581.
8. Klendshoj, N. C., Feldstein, M., and Sprague, A. Determination of 17 -ketosteroids in urine. J. clin. Endocr. 1953, 13, 922.

9. Silber, R. H., and Porter, C. C. The determination of 17,21-dihydroxy-20-ketosteroids in urine and plasma. J. biol. Chem. 1954, 210, 923.

10. Dalgliesh, C. E. The 5-hydroxyindoles. Advanc. clin. Chem. 1958, 1, 193.

11. Sobel, C., and Henry, R. J. Determination of catecholamines (adrenalin and noradrenalin) in urine and tissue. Amer. J. clin. Path. 1957, 27, 240.

12. Forsham, P. Personal communication.

13. Laidlaw, J. C., Jenkins, D., Reddy, W. J., and Jakobson, $T$. The diurnal variation in adrenocortical secretion. J. clin. Invest. 1954, 33, 950.

14. Raab, W. Abnormal suprarenal discharges in angina pectoris and their control by $\mathrm{X}$-ray therapy. J. clin. Endocr. 1941, 1, 977.

15. Raab, W., and Gigee, W. Total urinary catechol excretion in cardiovascular and other clinical conditions. Circulation 1954, 9, 592.

16. Gazes, P. C., Richardson, J. A., and Woods, E. F. Plasma catechol amine concentrations in myocardial infarction and angina pectoris. Circulation 1959, 19, 657.

17. Elmadjian, F., Hope, J. M., and Lamson, E. T. Excretion of epinephrine and norepinephrine under stress. Recent Progr. Hormone Res. 1958, 14, 513.

18. von Euler, U. S., Gemzell, C. A., Levi, L., and Ström, G. Cortical and medullary adrenal activity in emotional stress. Acta endocr. (Kbh.) 1959, $30,567$.

19. von Euler, U. S. Distribution and metabolism of catechol hormones in tissues and axones. Recent Progr. Hormone Res. 1958, 14, 483. (See Discussion, p. 508.)

20. Kaplan, A., Jacques, S., and Gant, M. Effect of long-lasting epinephrine on serum lipid levels. Amer. J. Physiol. 1957, 191, 8.

21. Sussman, K. E., Shafrir, E., and Steinberg, D. Mobilization of lipids by epinephrine. Circulation 1958, 18, 486.

22. White, J. E., and Engel, F. L. A lipolytic action of epinephrine and norepinephrine on rat adipose tissue in vitro. Proc. Soc. exp. Biol. (N. Y.) 1958, 99, 375.

23. Schotz, M. C., and Page, I. H. Effect of norepinephrine on plasma non-esterified fatty acids (NEFA) and the release of NEFA from rat epididymal adipose tissue. Fed. Proc. 1959, 18, 139.

24. Anitschkow, N. Über experimentell erzeugte Ablagerungen von anisotropen Lipoidsubstanzen in der Milz und im Knochenmank. Beitr. path. Anat. 1914, 57, 201.

25. Danisch, F. Die sympathischen Ganglien in ihrer Bedeutung für die Cholesterinsklerose des Kaninchens. Experimentelle Untersuchungen. Beitr. path. Anat. 1928, 79, 333. 